Musées, Patrimoine et Culture scientifiques et techniques

$171 \mid 2017$

mai-juin 2017

\title{
Faire de la culture scientifique, technique et industrielle une priorité
}

Samuel Cordier, Sylvane Casademont et Nicolas Ngo

\section{OpenEdition \\ Journals}

Édition électronique

URL : http://journals.openedition.org/ocim/1802

DOI : $10.4000 /$ ocim. 1802

ISSN : 2108-646X

\section{Éditeur}

OCIM

Édition imprimée

Date de publication : 1 mai 2017

Pagination : $42-45$

ISSN : 0994-1908

Référence électronique

Samuel Cordier, Sylvane Casademont et Nicolas Ngo, « Faire de la culture scientifique, technique et industrielle une priorité », La Lettre de l'OCIM [En ligne], 171 | 2017, mis en ligne le 01 mai 2018 consulté le 20 avril 2019. URL : http://journals.openedition.org/ocim/1802 ; DOI : 10.4000/ocim.1802

Ce document a été généré automatiquement le 20 avril 2019.

Tous droits réservés 


\title{
Faire de la culture scientifique, technique et industrielle une priorité
}

\author{
Samuel Cordier, Sylvane Casademont et Nicolas Ngo
}

Samuel Cordier : Pourquoi ce début d'année a été choisi pour finaliser et remettre ce rapport?

Sylvane Casademont : Nous n'avons pas choisi de remettre le rapport deux mois avant la fin de la mandature, même si je comprends que les gens se posent la question. La stratégie nationale a été élaborée par le Conseil national de la culture scientifique technique et industrielle (CSNCSTI), modifié par le décret de juillet 2014. Or la nomination des membres n'a pu être effective qu'en 2015. Le Conseil a tenu sa première réunion à l'automne 2015. Comme la date de fin de mandat des membres du CSNCSTI était avril 2017, nous avons eu un peu plus d'un an pour élaborer la stratégie et en rédiger le texte. C'était difficile de mettre moins de temps !

Nicolas Ngo : Depuis novembre 2015, en l'espace de 14 mois, le conseil s'est réuni cinq fois. Sylvane Casademont et Aliette Armel-Besnier, sous l'impulsion de Guillaume Houzel, alors conseiller de la ministre en charge de la CSTI, avaient bien préparé le terrain en recevant les acteurs, en cartographiant le champ, en rédigeant un certain nombre de propositions. Ce travail de fond nous a permis d'être réactifs au moment où le conseil a été effectivement mis en place. Ensuite, remettre un livre blanc ou une SNCSTI durant une année électorale, c'est un calendrier qui permet aussi de le diffuser auprès des différentes équipes de campagne, pour demander d'indexer la CSTI au budget de la recherche.

S. Co. : La Stratégie nationale de culture scientifique technique et industrielle (SNCSTI) s'inscrit dans la continuité de la loi Enseignement supérieur et Recherche du 22 juillet 2013 et du décret du 2 juillet 2014, renouvelant le CNCSTI. En quoi va t-elle plus loin ?

S. Ca. : La loi de 2013 décentralise aux Régions "sous réserve des missions de l'État", le suivi des actions de culture scientifique technique et industrielle. Elle instaure une gouvernance fondée sur la subsidiarité : aux acteurs, les actions et les projets ; aux 
Régions, la coordination des acteurs de leurs territoires ; à l'État, le soin de donner un cap, des objectifs communs, d'indiquer comment la CSTI s'inscrit dans les grandes priorités de l'État et de la Recherche. Si un article de la loi Fioraso indique que "la culture scientifique technique et industrielle doit être incluse dans la stratégie nationale de recherche", aucun ne précise qu'elle doit faire l'objet d'une stratégie nationale propre. Mais rien ne l'interdit non plus.

$\mathrm{Au}$ printemps 2013 en rencontrant et écoutant les acteurs de terrain, les CCSTI, les associations d'éducation populaire, des représentants des muséums, des responsables d'organismes de recherche, nous avons souhaité montrer que la CSTI n'est pas simplement une émanation ou un "sous-produit" de la recherche, mais un secteur vaste qui inclut à des titres divers des représentants de la société, des associations et des institutions. Il nous est donc apparu que, si la recherche est nécessaire, elle n'est pas suffisante pour faire sens au niveau de la culture scientifique. Nous avons donc proposé au Cabinet d'élaborer une stratégie nationale spécifique pour la culture scientifique.

S. Co. : Il existe selon vous un déficit de reconnaissance de la culture scientifique ? Même si beaucoup de choses ont été faites ces dernières décennies.

S. Ca. : Depuis bientôt cinq années que je travaille sur ce domaine, j'observe que la CSTI est mal connue du grand public et se connaît insuffisamment elle-même. En France, elle est connue par les deux à trois millions de personnes qui fréquentent la Fête de la Science, auxquelles vous ajoutez deux millions de personnes, mais ce sont peut-être les mêmes, qui ont pratiqué la culture scientifique dans d'autres lieux. Les acteurs me disent depuis 5 ans "nous ne nous connaissons pas forcément les uns les autres, nous ne sommes pas suffisamment connus ou reconnus". De plus, nous connaissons insuffisamment nos publics. C'est pourquoi le cinquième forum national l'année dernière a été organisé sur la thématique "Publics, territoires, médiation : quelle ambition pour la CSTI ?". Tout cela demande à être étudié, objectivé. Il n'existe pas forcément les données adéquates. C'est la raison pour laquelle il est nécessaire d'œuvrer à une connaissance et reconnaissance des acteurs. Et que cela figure précisément dans l'orientation 1 de la SNCSTI "Connaissance et reconnaissance des acteurs de la CSTI en France".

N. N. : Il nous arrive également de constater que certains chercheurs ont une connaissance toute relative de ce qu'est la culture scientifique aujourd'hui. Nous avons donc encore du travail à accomplir !

\section{S. Co. : Dans quel contexte s'est construite cette stratégie?}

N. N. : Nous étions dans le contexte post attentats 2015. Ces événements malheureux ont clairement cristallisé une prise de conscience, et une volonté de réfléchir et de formaliser ce que les professionnels sentaient de façon diffuse. C'est une des raisons pour lesquelles cette première stratégie nationale est porteuse d'une réelle ambition politique, au sens noble du terme.

S. Ca. : Cette stratégie est la compilation, pour une bonne partie, de ce qu'ont souhaité les acteurs. Presque toutes les orientations viennent du terrain. Prenons un exemple : celui du numérique. Le rapport Chicoineau ${ }^{2}$ avait identifié un besoin, le Programme investissement d'avenir (PIA) montre, surtout avec Inmédiats ${ }^{3}$, que le numérique est un champ qui mérite d'être investit et travaillé par les acteurs de la CSTI. Mais aussi, comme le rappelait Nadège Bouvard, directrice du réseau des médiathèques de Clamart, la fracture numérique existe bel et bien. Certaines personnes n'ont pas du tout accès au numérique ou ne savent pas utiliser Internet. Donc, si nous souhaitons nous adresser 
par le numérique à des populations défavorisées, il faut d'abord se préoccuper de leur équipement, et de leur capacité à l'utiliser ! Autre exemple, concernant le débat public et la question de la démarche scientifique : l'Amcsti $^{4} \mathrm{a}$ été la première à débattre de cette question en organisant la journée "Science, culture et croyance : comment en parler ?" l'année dernière. Je me souviens que des témoignages des médiateurs au cours de cette journée. Quand des gens à qui vous présentez l'évolution des espèces vous répondent : "vous descendez peut être du singe mais pas moi", "la science c'est une forme de croyance, la religion en est une autre, chacun croit ce qu'il veut"... Comment fait-on ? Comment la science, les scientifiques, peuvent-ils apporter leur contribution pour résoudre des problèmes fondamentaux sur lesquels les citoyens s'interrogent ? Et comment les médiateurs qui souhaitent populariser cette science, la partager et la faire aimer, peuvent-ils contribuer à battre en brèche les opinions, les faits alternatifs, les croyances ? Le fait de poser ces questions, à propos de la culture scientifique, nous a ouvert des horizons et donné le fil conducteur pour définir le sens, l'ambition de la stratégie.

S. Co. : Quelles sont les opérations envisagées par les acteurs de la CSTI pour accompagner la mise en œuvre de la stratégie nationale?

S. Ca. : Il faut d'abord la faire connaître. C'est pourquoi le 9 mars dernier, pour le lancement de la SNCSTI, nous avons réuni au Palais de la découverte les principaux acteurs issus des Régions. Nous souhaitons également intervenir dans les forums régionaux, si nous y sommes invités, pour faire un point ou échanger sur la mise en œuvre de cette stratégie.

Ensuite, l'essentiel : comment mettre en œuvre cette stratégie sur le terrain ? Quatre vingt pour cent, je pense, de la stratégie était déjà mise en œuvre au quotidien par tous les acteurs avant d'être publiée. Ce n'est pas la peine de s'escrimer à entrer dans les orientations, ils sont déjà dedans ! S'il existe une attente du CNCSTI, ce serait que les acteurs lancent davantage de projets en commun, en coordination pour leur donner davantage de visibilité et de force. Qu'ils s'emparent du document pour donner un sens ou un partage collectif à leurs actions. La stratégie peut devenir ainsi leur outil de travail. Mais surtout, qu'elle ne reste pas un rapport sur une étagère !

N. N. : Ce qui est remarquable, c'est que les acteurs de la CSTI s'inscrivent effectivement dans la logique de la stratégie, parfois même "inconsciemment". La SNCSTI constitue ainsi un outil de reconnaissance et de légitimation de leur action auprès des politiques locales.

S. Co. : La question des moyens de la mise en œuvre se pose aussi.

S. Ca. : Un aspect positif est que cette stratégie a fait prendre conscience au niveau de l'Etat, de l'importance du champ de la culture scientifique, du rôle qu'elle doit jouer visà-vis des enjeux sociétaux, mais aussi du nombre et de la multiplicité des acteurs. Aujourd'hui, cette prise de conscience se traduit par le souhait que l'État réinvestisse dans le champ de la CSTI, non pas à la place des Régions ou pour supporter seul le poids du domaine, mais pour faire effet de levier et construire des outils au service de toute la communauté, comme des plateformes de partage et d'échanges, ou des outils d'évaluation. Nous devons en effet pouvoir démontrer que la culture scientifique, contribue à faire évoluer les mentalités.

Nous n'avons pas parlé de la culture industrielle et c'est aussi un enjeu. Cette stratégie doit faire évoluer aussi les mentalités par rapport à l'économie, l'entreprenariat, le développement et l'innovation et travailler avec l'enseignement technologique et les entreprises qui offrent des contrats d'apprentissage. Si de l'argent est remis dans l'apprentissage, il faudrait que la culture technique et industrielle puisse aussi œuvrer dans le même sens. 
Ainsi, le Livre Blanc de l'Enseignement Supérieur et de la Recherche ${ }^{5}$ a bien souligné qu'il ne suffit pas de maintenir le budget de la recherche, mais il faut réinvestir dans ce domaine. La culture scientifique, dont la stratégie fait partie du Livre Blanc, doit obéir aux mêmes règles, car elle est un des bras armé de la recherche vers la société.

S. Co. : La SNCSTI peut donc être considérée comme un prolongement de la recherche vers la société?

S. Ca. : C'est cela. Si l'innovation technologique est envisagée comme le débouché de la recherche vers l'industrie ou l'économie, la culture scientifique peut être considérée comme le débouché de la recherche vers la société et au service de la démocratie. Il serait donc naturel que le budget de la recherche participe au financement de la CSTI en tant que telle. Les projets européens le font systématiquement, une partie du budget alloué par ce type d'appels à projets doit être consacrés au $\mathrm{RRI}^{6}$, au retour sur la société.

Le CNCSTI propose donc que l'État consacre un pourcentage du budget de la recherche à la CSTI. L'idéal serait d'obtenir pour la CSTI la même somme que celle du PIA 1 Égalité des chances, soit environ 100 millions d'euros sur cinq ans. Tel est l'objectif du Conseil Aucune décision n'a été prise. Il faut continuer à convaincre, mais aussi mettre en avant des arguments solides.

Ceci étant, il ne s'agit pas de remplacer les Régions ni d'empêcher les acteurs d'essayer de trouver de l'argent de toutes les manières possibles, y compris en ayant peut-être recours au crowdfunding. Mais l'idée du CNCSTI est qu'il faut réinvestir de l'argent public au niveau national pour que l'État apporte sa contribution à la mise en œuvre d'orientations stratégiques prioritaires. Plus il y aura d'argent dans la CSTI, mieux nous nous porterons.

S. Co. : La création d'une agence ou d'une fondation dont la mission serait de trouver d'autres sources de revenus que les financements publics a aussi été évoquée.

S. Ca. : C'est ce qui restera à déterminer si nous avons des financements. Le PIA a fait gérer cet argent par l'Agence nationale de rénovation urbaine (Anru) qui n'est peutêtre pas la plus adaptée pour des actions de CSTI. Quelles sont les agences existantes qui pourraient gérer ce type de crédit ? Parmi les agences d'État, il y a l'Agence nationale de la recherche (ANR), mais ce n'est pas sa vocation. C'est pourquoi le CNCSTI se demande si, pour gérer ces fonds, il ne faudrait pas mettre en place une agence nationale, qui pourrait effectivement lancer des projets et gérer un ensemble de missions nationales. Mais l'époque n'est pas à la création de structures. Il faudra sans doute imaginer d'autres solutions. Nous y travaillons.

S. Co. : Parmi les chantiers envisagés, il y en a un qui concerne les indicateurs et des outils d'évaluation

S. Ca. : Parmi les cinq orientations stratégiques figure en effet "L'évaluation de la stratégie", dans laquelle l'Ocim peut jouer un rôle important. Mais il y a beaucoup d'autres chantiers ! Je peux prendre aussi l'exemple de l'orientation "Numérique, connaissance, impact et usage du numérique", pour laquelle nous pourrons mobiliser l'Institut national de recherche en Informatique et automatique (Inria). Autre exemple, l'orientation "Débat démocratique et appui aux politiques publiques", avec l'action "Développer la formation et l'information des décideurs, entrepreneur(e)s et représentant(e)s de la sociétés civile, élu(e)s nationaux et locaux" pour lesquelles l'Institut des hautes études pour la science et la technologie (IHEST) pourrait être 
sollicité, tout comme pour l'action "Constituer un réseau de citoyennes et de citoyens concernés ou engagés". L'association des auditeurs de l'IHEST, qui regroupe les 400 cadres formés depuis 10 ans à l'IHEST, peut constituer une "réserve citoyenne pour la science", pour faire un parallèle avec la réserve citoyenne de l'Éducation nationale . Et, dans le cadre de l'orientation "Culture technique industrielle et innovation", il y a clairement des actions à mener avec les syndicats professionnels, les think tank ou les structures qui permettent de réunir des industriels. Aussi, la directrice générale de l'enseignement scolaire a présenté récemment la SNCSTI aux Recteurs d'Académie et les a appelés à se mobiliser, et a proposé d'intégrer la CSTI dans les Écoles supérieures du professorat et de l'éducation (Espe), c'est-à-dire dans les formations initiales des enseignants. C'est très important que le message qui porte la SNCSTI soit porté au plus haut niveau de l'Éducation nationale vers les acteurs.

S. Co. : Le CNCSTI sera-t-il renouvelé pour une période de cinq années ? Et quel est le calendrier de ses prochaines actions ?

S. Ca. : Le décret qui prolonge le conseil national de cinq ans est sorti le 24 avril. Les prochaines échéances sont précisément liées au financement, et à la mise en place du Nouveau Conseil national de la CSTI par nos deux ministres. Nous avons terminé une première phase de notre travail. Une nouvelle époque s'ouvre.

\section{La Stratégie nationale de culture scientifique, technique et industrielle}

Conçue pour 5 ans, la Stratégie nationale de culture scientifique, technique et industrielle (SNCSTI) est intégrée au Livre Blanc de l'Enseignement supérieur et de la Recherche et sera évaluée tous les deux ans par le Parlement. Son objectif : éclairer les citoyen(ne)s et leur donner les moyens de renforcer leur curiosité, leur ouverture d'esprit et leur esprit critique. Cette Stratégie nationale se structure autour de quatre thématiques transversales ("Égalité femmes/ hommes" ; "Changement climatique et développement durable" ; "Europe" ; "Histoire des sciences et des techniques") fondées sur les priorités de l'État et intègre cinq orientations stratégiques ("Connaissance et reconnaissance des acteurs" ; "Numérique : connaissance et usages" ; "Débats démocratique et appui aux politiques publiques" ; "Démarche scientifique partagée par la société" ; "Culture scientifique, industrielle et technique") visant à renforcer la CSTI.

\section{NOTES}

1. Le rapport est téléchargeable sur www.enseignementsup-recherche.gouv.fr.

2. Chicoineau, L. Partager les cultures scientifique, technique et industrielle à l'ère du numérique. 2015, $92 \mathrm{p}$.

3. Lauréat du Programme des Investissements d'Avenir, Inmédiats est porté par six centres de sciences Cap Sciences (Bordeaux Aquitaine), l'Espace des Sciences (Rennes Bretagne), La 
Casemate (Grenoble Agglomération), Relais d'sciences (Caen Basse-Normandie), Science Animation (Toulouse Midi-Pyrénées) et Universcience (Paris Île-de-France).

4. Association des musées et centres pour le développement de la culture scientifique technique et industrielle (Amcsti).

5. Remis le 31 janvier 2017 à la ministre de l'Éducation nationale, de l'Enseignement supérieur et de la Recherche, et au secrétaire d'État, Le Livre Blanc de l'Enseignement supérieur et de la Recherche, définit la stratégie de la France dans le domaine de l'enseignement supérieur et de la recherche et précise les mesures mises en œuvre dans le cadre de cette stratégie ainsi que les moyens nécessaires.

6. Responsible Research and Innovation (concept de Recherche et/ou d'Innovation Responsables).

INDEX

Mots-clés : Stratégie nationale, CSTI

\section{AUTEURS}

SAMUEL CORDIER

Directeur de l'Ocim

\section{SYLVANE CASADEMONT}

Directrice de cabinet du Directeur général de la recherche et de l'innovation

\section{NICOLAS NGO}

Adjoint au chef du département de la culture scientifique et des relations avec la société, au ministère de l'Éducation nationale, de l'Enseignement supérieur et de la Recherche 